\title{
Graft Polymerization of Dimethylacrylamide onto Polysilsesquioxane Containing Mercapto Groups
}

\author{
Shin-ichi Yamamoto, Tadahito Shimada, Atsuko Kimura, ${ }^{*}$ \\ Toshio SugIZAKI, ${ }^{*}$ and Osamu MORIYA ${ }^{\dagger}$ \\ Department of Applied Chemistry, National Defense Academy, Hashirimizu, Yokosuka 239-8686, Japan \\ ${ }^{*}$ Research Laboratory, Lintec Co., Nishiki-cho, Warabi 335-0005, Japan
}

(Received April 30, 2004; Accepted June 17, 2004; Published September 15, 2004)

\begin{abstract}
Polysilsesquioxane having phenyl and mercapto groups (PMPSQ) was prepared from (3-mercaptopropyl)trimethoxysilane (MTMS) and phenyltrimethoxysilane (PTMS) by co-condensation under basic conditions. The polymerization utilizing mercapto groups on PMPSQ with dimethylacrylamide (DMAA) under UV irradiation was shown to be a simple and convenient procedure for the formation of the graft polymer (PMPSQ- $g$-PDMAA). In the photo-initiated radical polymerization, no gelation was observed. The introduction of hydrophilic graft chain was effective to transform the hydrophobic polysilsesquioxane PTMS into the amphiphilic PMPSQ- $g$-PDMAA, which was evaluated by the measurements of contact angle. [DOI 10.1295/polymj.36.761]

KEY WORDS (3-Mercaptopropyl)trimethoxysilane / Phenyltrimethoxysilane / Graft Polymerization / Photopolymerization / Amphiphilic Polysilsesquioxane /
\end{abstract}

In a previous work of our laboratory, the preparation of grafted derivative of ladder-like polysilsesquioxane by the use of photoiniferter was investigated from the interests in a new kind of hybrid material. ${ }^{1}$ The grafted polysilsesquioxanes, soluble in usual organic solvents, are expected to show some specific properties based on the inorganic and organic polymeric components with the structural peculiarity. These should expand the utilities of polysilsesquioxane, which are demonstrated in various investigations concerning electrical, optical, mechanical, and chemical applications of polysilsesquioxanes. ${ }^{2,3}$ Furthermore, the expectation is supported by the recent studies on the introduction of polymeric components onto the related polysiloxane compounds such as silica, zeolite, and clay to modify or improve the properties. ${ }^{4-8}$ For the effective grafting, the living polymerization techniques via radical species such as atom transfer radical polymerization process $^{9-12}$ and the use of photoiniferter ${ }^{13}$ are appropriately utilized in the recent investigations. These living polymerization techniques contribute to minimize the formation of free chains, control the chain length, and the easy formation of block copolymer chains. However, the use of a metal/ligand complex or the introduction of dithiocarbamate group requires somewhat complicated treatments. On the other hand, thiol compound has been well known as a representative chain transfer reagent in radical polymerizations. ${ }^{14-16}$ Therefore, the radical process utilizing mercapto group has been reported as another efficient procedure to introduce polymeric chains on silica surface. ${ }^{17,18}$ Furthermore, very recently, such classical role of thiols is applied to the polymerization of methyl methacrylate without an initiator. ${ }^{19}$ In the graft polymerization onto polysilsesquioxane, mercapto group is expected to be the effective initiating site. In addition, the polysilsesquioxane containing mercapto group is easily obtained by the use of commercially available (3-mercaptopropyl)trimethoxysilane (MTMS). These will be the essential factors of a simple and practical procedure for the grafting onto polysilsesquioxane. However, such free radical process may result in the formation of gels via a radical coupling termination.

In line with the considerations mentioned above, we have started the investigations to examine and know the limitation of the procedure utilizing mercapto group for the graft polymerization onto the ladder-like polysilsesquioxane. Further, the introduction of hydrophilic components leading to an amphiphilic hybrid material is intended as another target. The resulting grafted product, containing both the hydrophobic and hydrophilic polymeric components, promises to show the interesting properties such as self-organizing and self-assembling, which should be utilized as a high performance copolymer. ${ }^{20,21}$ In this work, as shown in Scheme 1, the graft polymerization from the polysilsesquioxane having both phenyl and mercaptopropyl groups (PMPSQ) with dimethylacrylamide (DMAA), being known to afford a hydrophilic polymer, was conducted under UV irradiation to prepare the hybrid graft product PMPSQ- $g$-PDMMA. In

${ }^{\dagger}$ To whom correspondence should be addressed (E-mail: moriyaos@nda.ac.jp). 


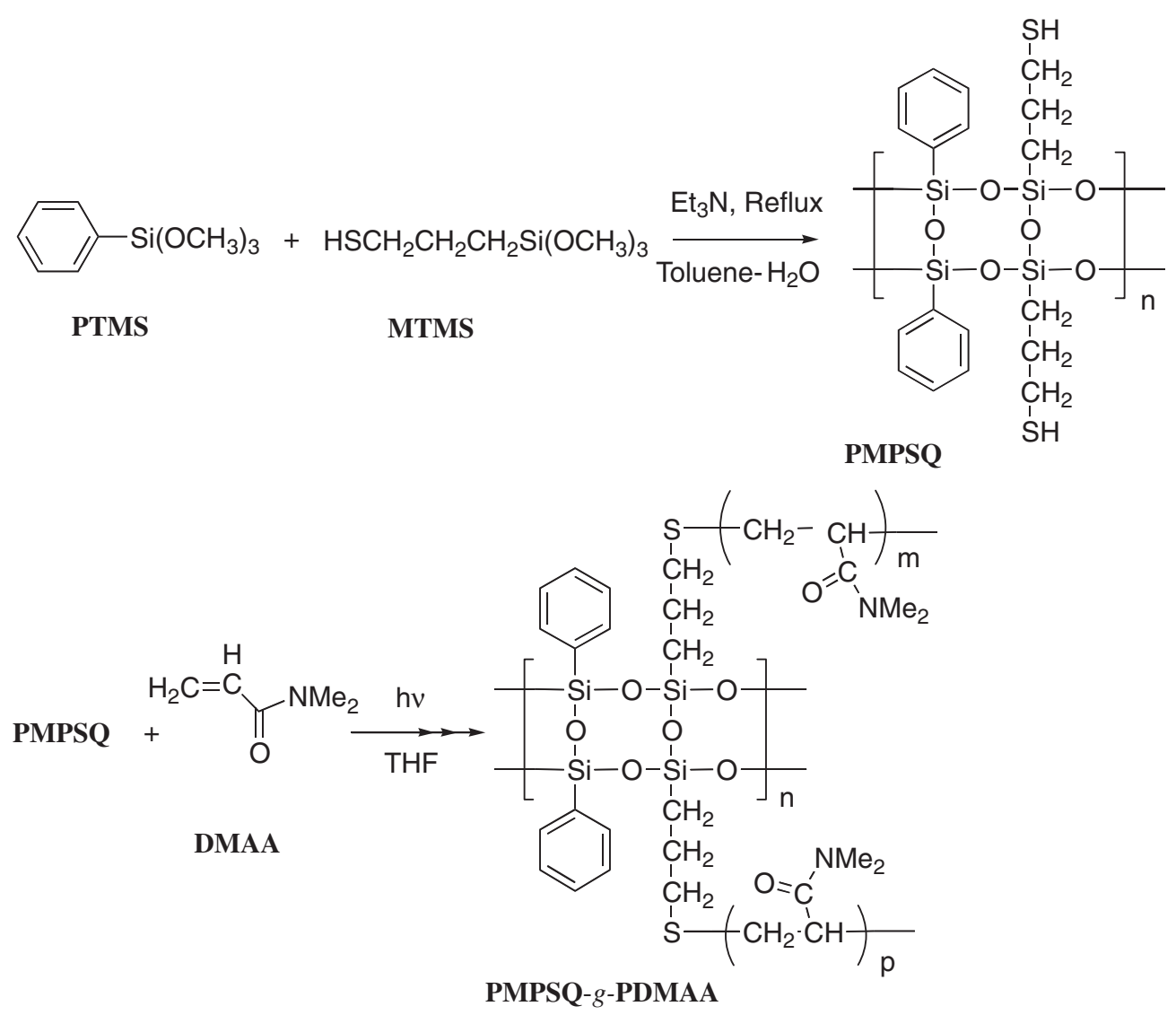

Scheme 1.

addition, the thermal behavior and the contact angle of PMPSQ- $g$-PDMMA were examined to obtain the fundamental information on the effects of the grafting.

\section{EXPERIMENTAL}

\section{General}

${ }^{1} \mathrm{H}$ NMR and ${ }^{13} \mathrm{C}$ NMR spectra were obtained on a JEOL FX-270 and JNM A-500 spectrometer in $\mathrm{CDCl}_{3}$. Solid-state $\mathrm{CP} / \mathrm{MAS}$ NMR measurements were carried out with samples in double air bearing $6 \mathrm{~mm}$ rotor of zirconia on a JEOL JNM ECA500 spectrometer, operating at $53.5 \mathrm{MHz}$ for ${ }^{29} \mathrm{Si}$. Magic angle spinning was performed at $5.6 \mathrm{kHz}$ spinning rate. The proton $90^{\circ}$ pulse was $4 \mu \mathrm{s}$ for ${ }^{29} \mathrm{Si}$ NMR measurements. Polydimethylsilane was the reference for ${ }^{29} \mathrm{Si}$ NMR spectra. IR spectra were recorded on a JASCO FT/IR 230. Gel permeation chromatographic (GPC) analysis was carried out on a Shimadzu LC-10VP chromatograph equipped with an evaporative light scattering detector. Three columns such as Shim-pack GPC-80MD, -804D, and -802D were connected in series and dimethylformamide (DMF) was used as the eluent. Calibration was performed using polymethylmethacrylate standards. THF employed for the reactions were refluxed over sodium metal and distilled. The monomers, dimethylacrylamide (DMAA) was dried with calcium hydride and distilled under reduced pressure before use. Other reagents including benzophenone were used as supplied from commercial sources.

\section{Preparation of PMPSQ}

A mixture of phenyltrimethoxysilane (PTMS, $4.00 \mathrm{~g}, 20.16 \mathrm{mmol})$ and MTMS (4.00 g, $20.36 \mathrm{mmol})$ with triethylamine $(0.20 \mathrm{~g}, 2.00 \mathrm{mmol})$ in toluene $(40 \mathrm{~mL})$ and water $(10 \mathrm{~mL})$ was refluxed for $24 \mathrm{~h}$. Organic phase was separated and washed with brine. The organic phase was concentrated by a rotary evaporator under reduced pressure and, then, methanol was added to the residual oil. The resulting wax-like oil insoluble in methanol was dried at room temperature for $24 \mathrm{~h}$ in a vacuum oven under reduced pressure $(<5 \mathrm{mmHg})$. PMPSQ (3.36g, 74\% yield from PTMS and 64\% yield from MTMS) was obtained as a solid. The contents of phenyl and mercapto groups were calculated from the peak areas observed in ${ }^{1} \mathrm{H}$ NMR spectrum, in which hexamethyldisiloxane was used as an internal standard; IR (KBr) 3450 (weak, OH), 3050 (weak, $-\mathrm{C}_{6} \mathrm{H}_{5}$ ), 2930 (medium, $-\mathrm{CH}_{2}-$ ), 2560 (weak, $\mathrm{SH}$ ), 1440 (medium), 1268 (medium), 1134 (strong, SiO), 1028 (strong, Si-O), 742 (medium), 700 (medium) 
$\mathrm{cm}^{-1} ;{ }^{1} \mathrm{H}$ NMR $\left(270 \mathrm{MHz}, \mathrm{CDCl}_{3}\right) \delta 0.45-0.95$ (br, $-\mathrm{Si}-\mathrm{CH}_{2}-$ ), 1.30 (br, $-\mathrm{S} \underline{\mathrm{H}}$ ), 1.45-1.85 (br, $-\mathrm{CH}_{2}-$ ), 2.25-3.00 (br, $-\mathrm{S}-\mathrm{CH}_{2}-$ ), 6.50-7.70 (br m, $-\mathrm{C}_{6} \underline{\mathrm{H}}_{5}$ ); ${ }^{13} \mathrm{C} \mathrm{NMR}\left(125 \mathrm{MHz}, \mathrm{CDCl}_{3}\right) \delta 12.40\left(\mathrm{Si}-\underline{\mathrm{CH}_{2}}-\right)$, $27.81\left(-\underline{\mathrm{CH}}_{2}-\mathrm{SH}, \mathrm{C}-\underline{\mathrm{CH}}_{2}-\mathrm{C}\right), 128.39\left(m, p-\overline{\mathrm{C}}_{6} \mathrm{H}_{5} \times\right.$ 3), $131.26\left(o-\mathrm{C}_{6} \mathrm{H}_{5} \times 2\right), 134.43\left(\mathrm{Si}-\mathrm{C}_{6} \mathrm{H}_{5}\right) ;{ }^{29} \mathrm{Si}$ $\mathrm{CP} / \mathrm{MAS}$ NMR $(53.5 \mathrm{MHz}) \delta-85.00$ to -73.00 , -73.00 to $-61.00,-61.00$ to $-53.00 ; M_{\mathrm{n}}=5700$, $M_{\mathrm{W}} / M_{\mathrm{n}}=2.07$; phenylsilyl unit $=4.46 \mathrm{mmol}$ equiv./ $\mathrm{g}$, mercapto unit $=3.85 \mathrm{mmol}$ equiv. $/ \mathrm{g}$.

\section{Typical Procedure for Grafting onto PMPSQ}

A solution of PMPSQ $(0.10 \mathrm{~g}, 0.45 \mathrm{mmol}$ equiv. of phenyl group and $0.39 \mathrm{mmol}$ equiv. of mercapto group), DMAA $(0.38 \mathrm{~g}, 3.83 \mathrm{mmol})$ in THF $(3.2 \mathrm{~mL})$ was introduced into a glass tube. The mixture was purged of air via three vacuum-argon cycles. Then, the mixture in the glass tube was irradiated at $20^{\circ} \mathrm{C}$ by using a RIKO RH400 UV lamp equipped with water jacket from a distance of $10 \mathrm{~cm}$ for $20 \mathrm{~h}$. The resulting solution was evaporated under reduced pressure and the residue was poured into diethyl ether to isolate insoluble wax-like oil. The oil was dried at room temperature for $24 \mathrm{~h}$ under reduced pressure $(<5 \mathrm{mmHg})$ to obtain the graft polymer (PMPSQ- $g$ PDMAA) as a solid $(0.37 \mathrm{~g}, 77 \%$ yield based on weight) (Table I, Run 5); IR (KBr) 3470, 2934, 1625 (C=O), 1495, 1400, 1260, 1140, 1095, $1045 \mathrm{~cm}^{-1} ;{ }^{1} \mathrm{H} \mathrm{NMR}\left(\mathrm{CDCl}_{3}, 500 \mathrm{MHz}\right) \delta 1.20(\mathrm{br}$, $\mathrm{m},-\underline{\mathrm{CH}}_{2}-$ ), 1.63-1.73 (br, m, $-\mathrm{C}_{2}-$ ), 2.15-2.48 (br, $-\underline{\mathrm{CH}}_{2}-$ ), 3.10 (br, $-\mathrm{CH}-\mathrm{N}-\mathrm{CH}_{3}$ ), 7.13-7.40 (br, $\left.-\mathrm{C}_{6} \underline{\mathrm{H}}_{5}\right) ;{ }^{13} \mathrm{C} \mathrm{NMR} \quad\left(125 \mathrm{MHz}, \mathrm{CDCl}_{3}\right) \quad \delta \quad 26.5$ $\left(-\underline{\mathrm{CH}}_{2}-\right), 35.8\left(\mathrm{NCH}_{3}\right), 36.3\left(\mathrm{NCH}_{3}\right), 37.1$ (- $\left.\underline{\mathrm{CH}}-\right)$, $125.2\left(-\underline{\mathrm{C}}_{6} \mathrm{H}_{5}\right), 127.8\left(-\underline{\mathrm{C}}_{6} \mathrm{H}_{5}\right), 133.7\left(-\underline{\mathrm{C}}_{6} \mathrm{H}_{5}\right), 174.6$ $(-\mathrm{C}=\mathrm{O})$; phenylsilyl unit $=1.18 \mathrm{mmol}$ equiv. $/ \mathrm{g}$ (97\% yield based on phenyl group), DMAA unit = $8.10 \mathrm{mmol}$ equiv./g $(78 \%$ yield based on the monomer).
Analogously, from the reaction of PMPSQ (0.10 g, $0.45 \mathrm{mmol}$ equiv. of phenyl group and $0.39 \mathrm{mmol}$ equiv. of mercapto group), DMAA (0.76 g, 7.67 mmol) in THF (6.4 mL), PMPSQ- $g$-PDMAA (0.51 $\mathrm{g}, 59 \%$ yield based on weight) was obtained (Table I, Run 6); phenylsilyl unit $=0.88 \mathrm{mmol}$ equiv./g $(100 \%$ yield based on phenyl group), DMAA unit $=8.60$ mmol equiv./g ( $57 \%$ yield based on the monomer).

Analogously, the polymerizations of PMPSQ with 6 equiv. of DMAA to mercapto group were carried out under various conditions (Figures 4 and 6; Table I, Runs 1-4).

\section{Thermal Analysis}

Preparation of Poly(dimethylacrylamide). A solution of DMAA $(10.00 \mathrm{~g}, 99.9 \mathrm{mmol}), \alpha, \alpha^{\prime}$-azobisisobutylonitrile $(\mathrm{AIBN})(0.20 \mathrm{~g}, 1.22 \mathrm{mmol})$ in toluene $(30 \mathrm{~mL})$ was introduced into a glass tube. The mixture was purged of air via three vacuum-argon cycles. The polymerization was carried out at $80{ }^{\circ} \mathrm{C}$ for $8 \mathrm{~h}$ under UV irradiation. The resulting solution was evaporated under reduced pressure and the residue was poured into diethyl ether. The precipitated solid was dried at room temperature for $24 \mathrm{~h}$ under reduced pressure $(<5 \mathrm{mmHg})$ to obtain the polymer (PDMAA) $\left(8.50 \mathrm{~g}, M_{\mathrm{n}}=13000, M_{\mathrm{w}} / M_{\mathrm{n}}=2.20\right)$.

PMPSQ, PMPSQ-g-PDMAA (Table I, Run 5) and PDMAA were employed for thermal degradation test by a thermogravimetric analyzer (DTG-60 by Shimadzu Corp.). The measurements were conducted with $10 \mathrm{mg}$ of the sample from room temperature to $800{ }^{\circ} \mathrm{C}$, in which a heating rate was $10^{\circ} \mathrm{C} \mathrm{min}^{-1}$ under nitrogen.

Contact Angle. A solution of PMPSQ or PMPSQ$g$-PDMAA (Table I, Run 5 or 6) in toluene was cast on poly(ethylene terephtalate) sheet and, then, removed toluene at $120^{\circ} \mathrm{C}$ for $1 \mathrm{~min}$. in an oven. Static contact angle was measured with deionized water

Table I. Preparation of PMPSQ- $g$-PDMAA from PMPSQ

\begin{tabular}{|c|c|c|c|c|c|c|}
\hline \multirow[b]{2}{*}{ Run } & \multicolumn{2}{|c|}{ Reaction conditions ${ }^{\mathrm{a}}$} & \multicolumn{4}{|c|}{ PMPSQ- $g$-PDMAA } \\
\hline & $\begin{array}{c}\text { Monomer } \\
\text { concentration } \\
{[\mathrm{M}]}\end{array}$ & $\begin{array}{c}\text { Ratio of } \\
\text { DMAA/SH } \\
\mathrm{mol} / \mathrm{mol}\end{array}$ & $\begin{array}{c}\text { Yield } \\
\%\end{array}$ & $\begin{array}{c}\text { Unit ratio of } \\
\text { DMAA } / \mathrm{SH}^{\mathrm{b}} \\
\text { mol eq./mol eq. }\end{array}$ & $M_{\mathrm{n}}^{\mathrm{c}}$ & $M_{\mathrm{w}} / M_{\mathrm{n}}^{\mathrm{c}}$ \\
\hline 1 & 0.3 & 6 & 73 & 4.2 & 12800 & 2.02 \\
\hline 2 & 0.6 & 6 & 75 & 4.3 & 12500 & 1.95 \\
\hline 3 & 1.2 & 6 & 80 & 4.7 & 13000 & 2.05 \\
\hline 4 & 2.3 & 6 & 86 & 4.9 & 13600 & 2.13 \\
\hline 5 & 1.2 & 10 & 77 & 7.8 & 21800 & 2.01 \\
\hline 6 & 1.2 & 20 & 59 & 11.3 & 28600 & 2.03 \\
\hline
\end{tabular}

${ }^{a}$ The polymerization of PMPSQ containing $3.85 \mathrm{mmol}$ eq. $/ \mathrm{g}$ of mercapto groups was conducted at $20^{\circ} \mathrm{C}$ in $\mathrm{THF}$ under UV irradiation for $10 \mathrm{~h} .{ }^{\text {b}}$ The molar ratio of DMAA unit and SH unit was calculated on the basis of integrals in the ${ }^{1} \mathrm{H}$ NMR spectra measured with hexamethyldisiloxane as an internal standard. The content of SH unit was estimated from the amount of phenyl group, the ratio of which and SH unit was assumed to be same to that in the starting PMPSQ. ${ }^{\mathrm{c}}$ Determined by GPC with polymethylmethacrylate standards. 
drops on the surface of the resulting sheet within $10 \mathrm{~s}$. at room temperature by a Face Contact Angle Meter (CA-D Type by Kyowa Interface Science Co., Ltd.).

\section{RESULTS AND DISCUSSION}

\section{Preparation of PMPSQ from PTMS and MTMS}

The polysilsesquioxane, PMPSQ, having phenyl and mercaptopropyl groups was prepared as the starting material in this work. Usually, the ladder-like poly(phenylsilsesquioxane), soluble in various organic solvents, could be obtained effectively as an easy handling solid under basic conditions from PTMS. ${ }^{22,23}$ On the other hand, the condensation using sole MTMS proceeded with gelation in the presence of a basic catalyst. Consequently, the introduction of phenyl groups was required to isolate the polysilsesquioxane containing mercaptopropyl groups as a solid soluble in usual organic solvents. Furthermore, the presence of phenyl groups was thought to be preferable in the ${ }^{1} \mathrm{H}$ NMR analyses of PMPSQ and the products, since the signal assigned to phenyl group was available as a standard for the calculation of the composition of several groups containing in the derivatives of PMPSQ. The co-condensation of PTMS and MTMS with a catalytic amount of triethylamine was carried out to prepare PMPSQ. The starting material, PMPSQ, contained the almost same molar ratio of phenyl and mercaptopropyl groups, was obtained in the good yield such as $74 \%$ as a wax-like oil insoluble in methanol. After drying under vacuum at room temperature, the wax-like oil changed to the easy handling solid. The composition of the groups in PMPSQ reflected the feed molar ratio of PTMS and MTMS. Such estimations of the yield and the contents of phenyl and mercapto groups were based on the proton ratios observed in the ${ }^{1} \mathrm{H}$ NMR spectral data.

In the ${ }^{1} \mathrm{H}$ NMR measurements of PMPSQ, hexamethyldisiloxane was used as an internal standard, the signal of which appeared at $0 \mathrm{ppm}$. As shown in Figure 1, the signals assigned to the protons for $-\mathrm{CH}_{2} \mathrm{CH}_{2} \mathrm{SH}$ were observed in the range from 1.30 to $1.85 \mathrm{ppm}$. Other protons showing the presences of $\mathrm{SiCH}_{2}-$ and benzene ring were detected around $0.9 \mathrm{ppm}$ and $7.6 \mathrm{ppm}$, respectively. From the proton ratios of these signals, the contents of $3.85 \mathrm{mmol}$ equiv./g of mercapto group and $4.46 \mathrm{mmol}$ equiv./g of phenyl group were estimated. In the spectrum of ${ }^{13} \mathrm{C}$ NMR, shown in Figure 2, the signals due to the groups mentioned above were also detected. The carbons assigned to $\mathrm{SiCH}_{2}-$ and $-\mathrm{CH}_{2} \mathrm{SH}$ were appeared at $12.40 \mathrm{ppm}$ and $27.81 \mathrm{ppm}$, respectively. The later signal also showed the presence of another methylene carbon as previously reported in the solid-state ${ }^{13} \mathrm{C}$ NMR measurements. ${ }^{24-26}$ The spectral data of ${ }^{29} \mathrm{Si}$

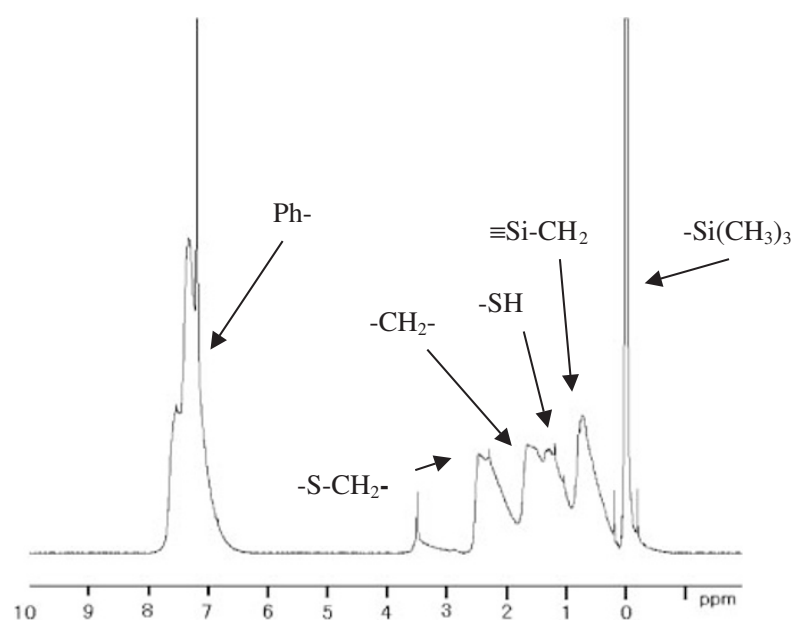

Figure 1. ${ }^{1} \mathrm{H}$ NMR spectrum $\left(\mathrm{CDCl}_{3}, 270 \mathrm{MHz}\right)$ of PMPSQ.

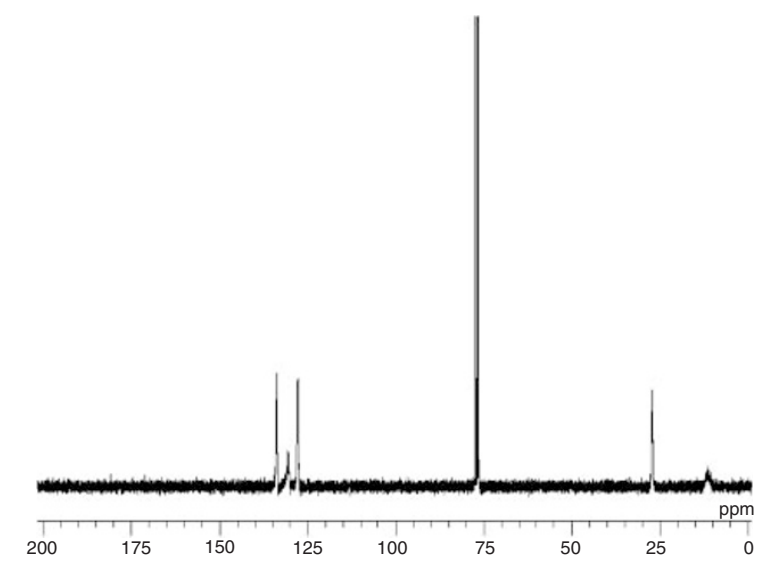

Figure 2. ${ }^{13} \mathrm{C}$ NMR spectrum $\left(\mathrm{CDCl}_{3}, 67.5 \mathrm{MHz}\right)$ of PMPSQ.

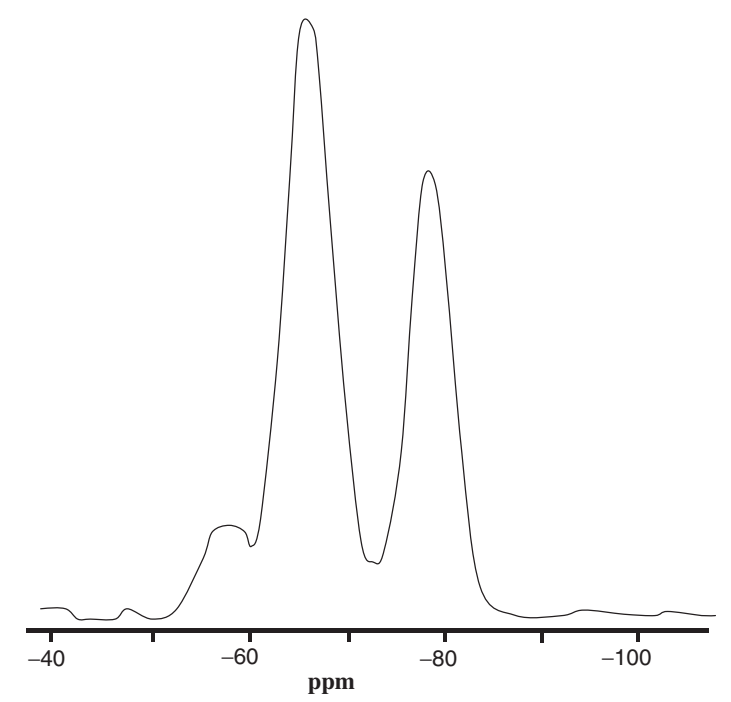

Figure 3. Solid-state ${ }^{29} \mathrm{Si}$ NMR spectrum of PMPSQ.

NMR of PMPSQ presented the information about the structure of polysiloxane backbone as shown in Figure 3 . The signal observed at $-78 \mathrm{ppm}$ demonstrated that the polymerized structure contained phenyl 
substituted silicon-oxygen $\mathrm{T}^{3}$ units. The signal at $c a$. $-66 \mathrm{ppm}$ seemed to contain the signals assigned to $\mathrm{T}^{2}$ units of phenyl substituted silicon and mercaptopropyl substituted $\mathrm{T}^{3}$ units. The presence of $\mathrm{T}^{2}$ units of mercaptopropyl silicon-oxygen was exhibited by the signal appeared at $-58 \mathrm{ppm}$. The reported spectral data of silica immobilized mercaptopropyl groups support those interpretations. ${ }^{7}$ Generally, the chemical shifts of $\mathrm{T}^{2}$ and $\mathrm{T}^{3}$ were reported to vary with the difference of structures and molecular weights. ${ }^{27,28}$ In the ${ }^{29} \mathrm{Si}$ NMR spectra of oligo- and poly(phenylsilsesquioxane), $\mathrm{T}^{3}$ silicone signal of oligocyclic one is observed at $-64.1 \mathrm{ppm}$ and that of polymerized one is at $-83.7 \mathrm{ppm} .{ }^{28}$ The later chemical shifts of the polysilsesquioxane, $M_{\mathrm{n}}$ of which is 53800 , is explained by the environmental homogeneity of each repeating unit. The chemical shift of PMPSQ, ca. $-78 \mathrm{ppm}$, was in the middle of those reported values. This may indicate that siloxane backbone of PMPSQ involve the regular ladder-like repeating units, cyclic units, and irregular silsesquioxane units. However, the assignments on the overlapped signals made it difficult to estimate the contents of two kinds of silicon having phenyl and mercaptopropyl group, respectively.

The IR spectrumof PMPSQ also supported the presences of phenyl and mercapto groups as shown in Figure 4. The absorption observed at $3025 \mathrm{~cm}^{-1}$ and weak absorptions around $1650 \mathrm{~cm}^{-1}$ in the spectrum were assigned to phenyl groups on the ladder-like siloxane backbone. The presence of mercapto groups was shown in the weak absorption observed at $2560 \mathrm{~cm}^{-1}$. The strong and broad absorption appeared around $1130 \mathrm{~cm}^{-1}$ was assigned to $\mathrm{T}^{2}$ and $\mathrm{T}^{3}$ units consisted of $\mathrm{Si}-\mathrm{O}$ bonds. These were reported to be observed at $1132 \mathrm{~cm}^{-1}$ and $1078 \mathrm{~cm}^{-1}$ in the IR spectra of poly(methylsilsesquioxane). ${ }^{29}$ The numberaverage molecular weights $\left(M_{\mathrm{n}}\right)$ and polydispercity

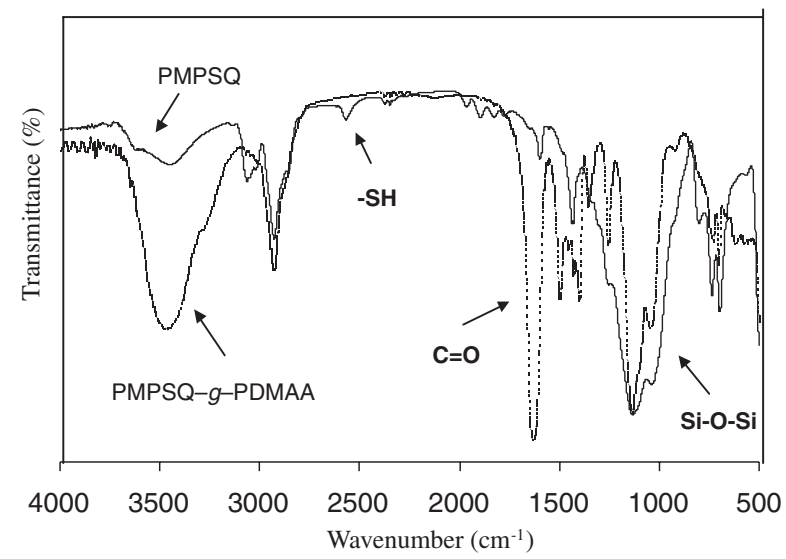

Figure 4. IR spectra of PMPSQ (-) and PMPSQ- $g$ PDMAA (…) (Table I, Run 5).

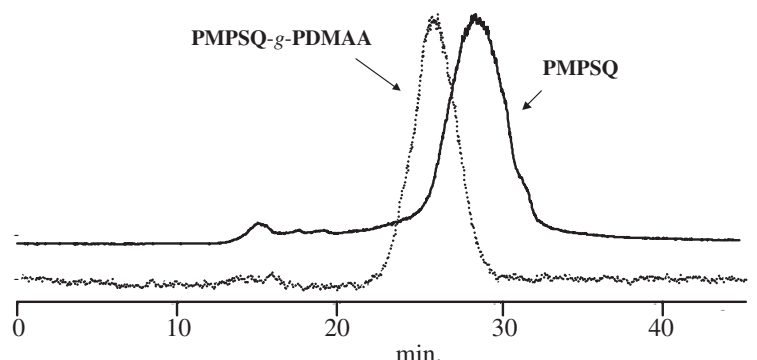

Figure 5. GPC traces for PMPSQ (-) and PMPSQ- PDMAA $(\cdots)$ (Table I, Run 5).

$\left(M_{\mathrm{w}} / M_{\mathrm{n}}\right)$ of PMPSQ estimated by GPC data were ca. 5700 and 2.07, respectively as shown in Figure 5. PMPSQ was soluble in diethyl ether, chloroform, benzene, acetone, THF, and DMF, but insoluble in methanol, $n$-hexane and water. Such PMPSQ was used as the starting material for the graft polymerization.

\section{Graft Polymerization}

The photopolymerization of DMAA with PMPSQ under UV irradiation using a high pressure merculy lamp at $25^{\circ} \mathrm{C}$ was carried out in THF with or without a catalytic amount of benzophenone or $\alpha, \alpha^{\prime}$-azobisisobutyronitrile (AIBN). The yields and $M_{\mathrm{n}} \mathrm{s}$ of the products were almost same under these conditions. The results suggested that this radical polymerization proceeded similarly to the reported one of methyl methacrylate, in which no initiator was employed. ${ }^{19}$ According to the report, the polymerization was speculated to begin from the step of polar hydrogen transfer from mercapto group to methyl methacrylate and, then, radical species were generated. The similar mechanism may be applicable to explain the efficient graft polymerization of DMAA in our polymerization system. From those results, the graft polymerizations in this work were examined without a photo-sensitizer or radical-initiator.

At first, the graft polymerization using six equiv. of DMAA to mercapto group in $1.2[\mathrm{M}]$ solution of THF was conducted, in which the conversions of DMAA were monitored by ${ }^{1} \mathrm{H}$ NMR spectra and the $M_{\mathrm{n}} \mathrm{s}$ of the corresponding graft polymer were by GPC. As shown in Figure 6, the plot of relative conversion of the monomer $v s$. time indicated that the conversion reached to $c a$. $80 \%$ after $6 \mathrm{~h}$. The linear line of the calculated $M_{\mathrm{n}} \mathrm{s}$ from the conversions, where the converted monomers were assumed to be grafted completely, was shown in Figure 7. The $M_{\mathrm{n}} \mathrm{s}$ of the graft polymer estimated by GPC also increased along with the calculated line, although the values became somewhat smaller in the region of higher conversions. The increase of polydispersities $\left(M_{\mathrm{w}} / M_{\mathrm{n}}\right)$ from $c a .1 .5$ to ca. 2.0 according to the increase of the conversions 


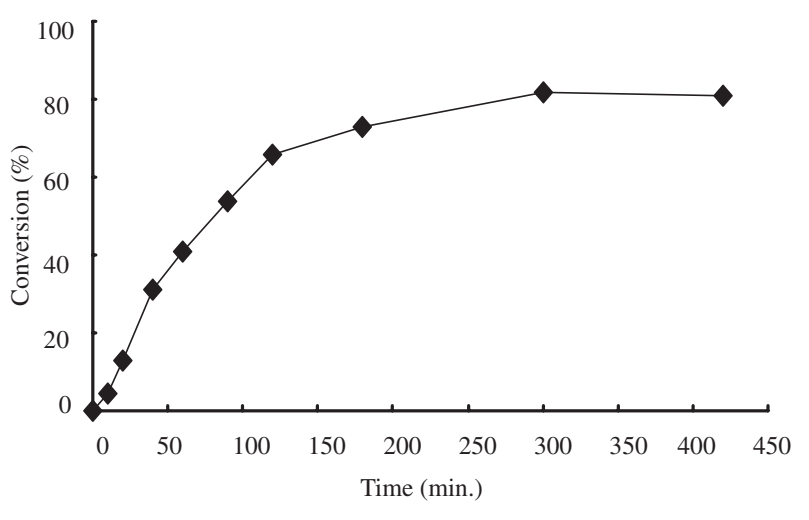

Figure 6. Time-conversion relation in graft polymerization of PMPSQ $(0.10 \mathrm{~g})$ using six equiv. of DMAA $(2.34 \mathrm{mmol})$ to mercapto group in THF $(2.0 \mathrm{~mL})$ at $20^{\circ} \mathrm{C}$ (Table I, Run 3$)$.

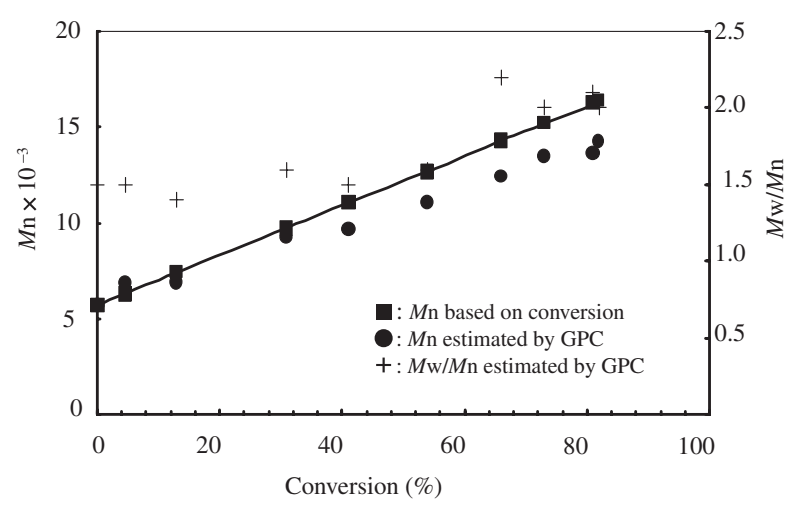

Figure 7. Conversion- $M_{\mathrm{n}}$ and $M_{\mathrm{w}} / M_{\mathrm{n}}$ relations in graft polymerization of PMPSQ ( $0.10 \mathrm{~g})$ using six equiv. of DMAA (2.34 mmol) to mercapto group in THF $(2.0 \mathrm{~mL})$ at $20^{\circ} \mathrm{C}$ (Table I, Run 3).

was observed. These results seemed to show that the monomer essentially grafted on PMPSQ. However, a slight deviation of $M_{\mathrm{n}} \mathrm{s}$ from the line of the calculated $M_{\mathrm{n}} \mathrm{s}$ may suggest the formation of the homopolymer of DMAA.

Next, the graft polymerizations were carried out under several different conditions. The results were shown in Table I. In the polymerizations, in which the concentrations of the monomer were adjusted to be in the range from $0.3 \mathrm{~mol} / \mathrm{L}$ to $2.3 \mathrm{~mol} / \mathrm{L}$, the yields increased from $75 \%$ to $86 \%$ as the increase of the concentrations (Table I, Runs 1-4). The values of $M_{\mathrm{n}}$ estimated by GPC were also increased according to the conversion from 12500 to 13600 . In the concentrations of 1.2 and $2.3 \mathrm{~mol} / \mathrm{L}$, the increase of the conversion and $M_{\mathrm{n}}$ of the resulting polymer was small. The other factor, which related to the efficiency of grafting or polymerization, was the molar feed ratio of mercapto group to the monomer. This was demonstrated by changing the feed molar ratios of DMAA to mercapto group (Table I, Runs 3, 5, and 6). In the cases using 6 and 10 equiv. of DMAA, the insoluble solid in diethyl ether was obtained in the analogous yields such as $77 \%$. However, the decrease of the yield, $59 \%$, was recorded in the use of 20 equiv. of DMAA. These yields were thought to relate directly with the incorporated monomer units. Because, the estimation based on the ${ }^{1} \mathrm{H}$ NMR spectral data indicated that PMPSQ unit was quantitatively recovered as the main chain of PMPSQ- $g$-PDMAA. This meant that the yields were essentially depended on the amount of polymerized DMAA. Furthermore, in GPC profiles of the starting PMPSQ and the products, one main peak was observed, respectively, and no additional peak was appeared after grafting. This supported the assumption mentioned above that DMAA was primarily incorporated into PMPSQ as the graft chain and the content of the homopolymer should be small even if it was formed. The GPC curves of PMPSQ- $g$ PDMAA (Table I, Run 5) and PMPSQ was shown in Figure 5. In these graft polymerizations, PMPSQ$g$-PDMAAs were isolated as the wax-like oil insoluble in diethyl ether, but soluble in water, methanol, acetone, chloroform, benzene, THF, and DMF. Furthermore, it was noteworthy that no cross-linked product was detected in such simple radical polymerization system.

The spectral data of IR and NMR supported the incorporation of the graft chains onto PMPSQ. In all the IR spectra of the grafted products, the broad absorptions around $1200 \mathrm{~cm}^{-1}$ assigned to $\mathrm{C}-\mathrm{O}$ bond were observed, where the absorption due to $\mathrm{Si}-\mathrm{O}$ bond might be included. The absorptions due to carbonyl group of the acrylamide polymer appeared clearly at $c a .1630 \mathrm{~cm}^{-1}$ in the spectra of the polysilsesquioxanes after grafting. The weak absorption at $2560 \mathrm{~cm}^{-1}$ assigned to SH group was disappeared in the spectra of PMPSQ- $g$-PDMAA. The spectra of PMPSQ and PMPSQ- $g$-PDMAA were shown in Figure 4. The presence of grafting components was, further, confirmed by the spectral data of NMR. In the ${ }^{1} \mathrm{H}$ NMR spectra of the grafting polysilsesquioxanes, the signals due to the protons of methylene and methine groups were detected in the range from $1.2-1.8 \mathrm{ppm}$. The methylene protons attached to $\mathrm{Si}$ was observed at $0.7 \mathrm{ppm}$ in the spectra of PMPSQ$g$-PDMAA, which was prepared from PMPSQ with six equiv. of DMAA. Other signals due to methyl protons of dimethylamino group and protons of benzene ring were appeared around $3.0 \mathrm{ppm}$ and $7.2 \mathrm{ppm}$, respectively. In the spectra used for the examination on the relationship of time vs. conversion, vinyl protons were detected in the region of $5.5 \mathrm{ppm}$ to $6.8 \mathrm{ppm}$. The proton ratios of the signals due to vinyl, phenyl, dimethyl, methylene, and methyl, were employed for the calculations of the contents of the monomer, the polymerized monomer, and polysilsesquioxane backbone, which were based on that of hex- 


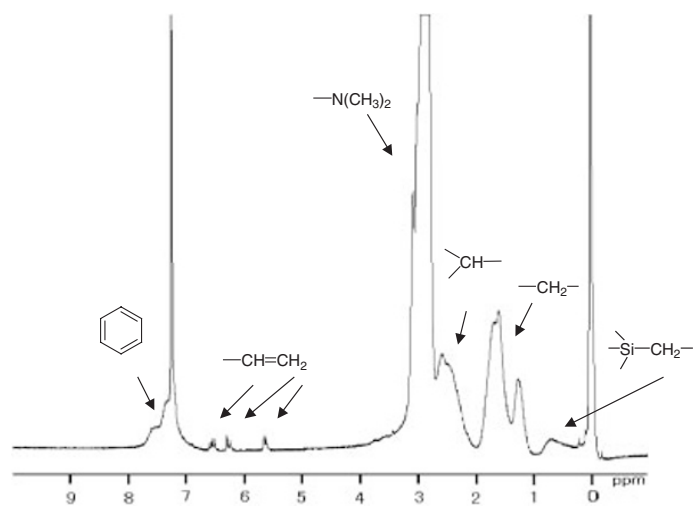

Figure 8. ${ }^{1} \mathrm{H}$ NMR spectrum $\left(\mathrm{CDCl}_{3}, 270 \mathrm{MHz}\right)$ of the mixture in the graft polymerization (Table I, Run 4).

amethyldisiloxane as an internal standard. An example of the spectra was shown in Figure 8. The existences of the monomer unit in the graft polysilsesquioxanes were also confirmed by the ${ }^{13} \mathrm{C}$ NMR spectra. The signals around $130 \mathrm{ppm}$ and $36 \mathrm{ppm}$ observed in all the spectra of the graft polymers were assigned to carbons of phenyl and dimethylamino group, respectively. The signal appeared around $178 \mathrm{ppm}$ exhibited the incorporation of carbonyl groups of the monomer units after grafting process.

The thermal stability of the graft polymer PMPSQ$g$-PDMAA (Table I, Run 5) was examined by TGA analysis and compared with those of poly(dimethylacrylamide) PDMAA and PMPSQ as shown in Figure 9. PDMAA was prepared by free radical polymerization, $M_{\mathrm{n}}$ of which was 13000 . In the measurements, conducted a heating rate of $10^{\circ} \mathrm{C} \mathrm{min}^{-1}$ under nitrogen, the obvious degradation of poly(dimethylacrylamide) chain began at $c a .380^{\circ} \mathrm{C}$ in all samples.

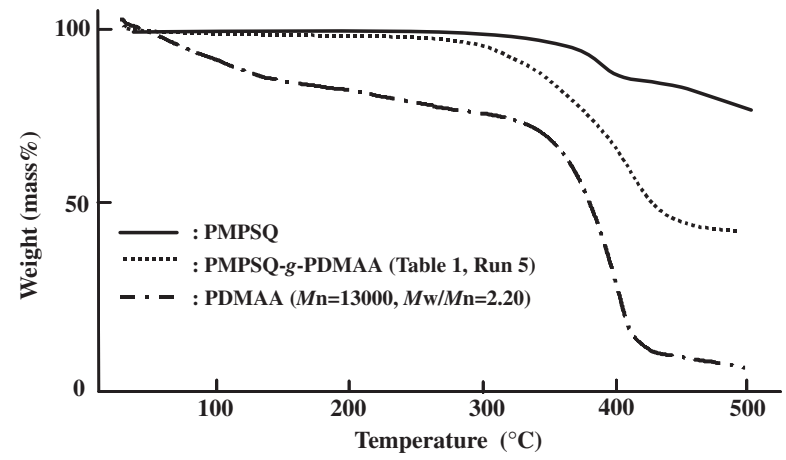

Figure 9. TGA curves of PMPSQ (-), PMPSQ- -PDMAA $(\cdots)$ (Table I, Run 5), and PDMAA (---).
In comparison with the weight loss until $300^{\circ} \mathrm{C}$, that of PMPSQ- $g$-PDMAA was $c a$. 5\%. In the case of PDMAA, the weight loss was $c a .25 \%$. Such results seem to demonstrate the improved heat-stability of the graft polysilsesquioxane PMPSQ- $g$-PDMAA. However, the tolerance of the grafting units to heat, caused by the combination with polysiloxane structure, was not so obvious. Since, the decrease of the weights of PMPSQ- $g$-PDMAA and PDMAA began at almost the same temperature such as $c a .170^{\circ} \mathrm{C}$.

The effect of incorporation of the hydrophilic graft chains was evaluated by the measurement of contact angle on the films obtained from PMPSQ- $g_{-}$ PDMAAs. The contact angles of the water drop and the surface, measured at room temperature within $10 \mathrm{~s}$, were shown in Table II. The hydrophobic PMPSQ provided the reasonable angle such as $98^{\circ}$. The contact angle of PMPSQ- $g$-PDMAA containing 7.8 monomer units to one mercapto group (Table I, Run 5) obviously decreased to be $18^{\circ}$. In the case of PMPSQ- $g$-PDMAA containing 11.3 monomer units (Table I, Run 6), the angle was $12^{\circ}$. Thus, the angle decreased with the increase of contents of the hydrophilic monomer units, although the accuracy of the values of PMPSQ- $g$-PDMAAs might be governed by an experimental error.

\section{CONCLUSIONS}

The ladder-like polysilsesquioxane containing phenyl and mercaptopropyl groups was employed as an effective starting material for the preparation of grafted hybrid polymer. From the results, mercapto groups in the polysilsesquioxane were revealed to be the effective initiation sites for the graft polymerization under UV irradiation. In the graft polymerizations of PMPSQ with DMAA, no formation of cross-linked product was detected. As an expected physical property, the graft polymers should show a better thermal stability in comparison with the homopolymer. This was not obviously demonstrated, but it might say at least that the weight-loss of PMPSQ- $g$-PDMAA was smaller below $300^{\circ} \mathrm{C}$ compared to that of the homopolymer PDMAA. The use of DMAA as the monomer to give the hydrophilic graft chains was successfully shown in the decreases of contact angle of PMPSQ- $g$-PDMAA. Thus, the introduction of the hydrophilic graft chains enabled the preparation of the

Table II. Contact angle of water on the surface of PMPSQ and PMPSQ- $g$-PDMAA

\begin{tabular}{lccc}
\hline \multicolumn{1}{c}{ Material } & PMPSQ & \multicolumn{2}{c}{ PMPSQ- $g$-PDMAA } \\
\hline $\begin{array}{l}\text { Molar unit ratio } \\
\text { of DMAA/SH }\end{array}$ & 0 & 7.8 & 11.3 \\
\hline Contact angle & $98^{\circ}$ & $18^{\circ}$ & (Table I, Run 6) \\
\hline
\end{tabular}


ladder-like polysilsesquioxane soluble in water. Such graft polymer is expected to be a widely utilized amphiphilic hybrid material showing a higher thermal stability and weather durability for a polymeric surfactant and the additive in paint and adhesive.

\section{REFERENCES}

1. O. Moriya, S. Yamamoto, T. Kumon, T. Kageyama, A. Kimura, and T. Sugizaki, Chem. Lett., 33, 224 (2004).

2. R. H. Baney and X. Cao, in "Silicon-Containing Polymers," R. G.Johns, W. Ando, and J. Chojnowski, Ed., Kluwer, Dordrecht, 2000.

3. C.-L. Chang and C.-C. M. Ma, J. Polym. Sci., Part A: Polym. Chem., 41, 1371 (2003).

4. K. Bridget and B. Vincent, Eur. Polym. J., 16, 1017 (1980).

5. B. M. Novak, Adv. Mater., 12, 675 (1993).

6. A. P. Alivisatos, Science, 271, 933 (1996).

7. C. Bartholome, E. Beyou, E. Bourgeat-Lami, P. Chaumont, and N. Zydowicz, Maclomolecules, 36, 7946 (2003).

8. H. Zhao, S. D. Argoti, B. P. Farrell, and D. A. Shipp, J. Polym. Sci., Part A: Polym. Chem., 42, 918 (2004).

9. D. Xiano and M. J. Wirth, Macromolecules, 35, 2919 (2002).

10. V. M. Litvinov, H. Barthel, and J. Weis, Macromolecules, 36, 4356 (2003).

11. J. Pyun, K. Matyjaszewski, J. Wu, G.-M. Kim, S. B. Chun, and P. T. Mather, Polymer, 44, 2739 (2003).

12. S. C. Hong, J.-F. Lutz, Y. Inoue, C. Strissel, O. Nuyken, and K. Matyjaszewski, Macromolecules, 36, 1075 (2003).

13. J. Bay, K.-U. Qiu, and Y. Wen, Polym. Int., 52, 853 (2003).

14. R. P. Steer and A. R. Knight, J. Phys. Chem., 72, 2145
(1968).

15. L. Lecamp, F. Houllier, B. Youssef, and C. Bunnel, Polymer, 42, 2727 (2001).

16. M. Horio, Y. Nishijima, T. Fujimoto, K. Ozawa, C. Kujirai, Y. Nakamura, and T. Kondo, Appl. Polym. Symp., 1971, 513.

17. M.-Y. Huang, R. Wu, and L.-R. Jiang, Polym. Bull., 9, 5 (1983).

18. N. Tsubokawa, T. Kimoto, and K. Koyama, Colloid Polym. Sci., 271, 940 (1993).

19. P. Xia, H. Cheng, and D. Van, J. Appl. Polym. Sci., 45, 579 (1992).

20. M. Anthonietti, S. Förster, J. Hartmann, and S. Oestreich, Maclomolecules, 29, 3800 (1996).

21. M. Sciborek, N. K. Gladkova, and J. Chojnowski, Polym. Bull., 44, 377 (2000).

22. Y. Matsubara, W. Konishi, T. Sugizaki, and O. Moriya, J. Polym. Sci., Part A: Polym. Chem., 39, 2125 (2001).

23. O. Moriya, M. Fukushima, T. Sugizaki, Y. Nakamura, and T. Endo, J. Polym. Sci., Part A: Polym. Chem., 40, 286 (2002).

24. E. F. S. Vieira, J. de A. Simoni, and C. Airoldi, J. Mater. Sci., 7, 2249 (1997).

25. I. M. El-Nahhal, J. J. Yang, I.-S. Chuang, and G. E. Maciel, J. Non-Cryst. Solids, 208, 105 (1996).

26. L. Mercier and T. J. Pinnavaia, Adv. Mater., 9, 500 (1997).

27. C. Ma and Y. Kimura, Polym. J., 34, 709 (2002).

28. E. Lippma, M. Magi, A. Samonson, G. Engelhardt, and A. G. Grimmer, J. Polym. Sci., Part A: Polym. Chem., 37, 1017 (1999).

29. N. Takamura, T. Gunji, H. Hatano, and Y. Abe, J. Polym. Sci., Part A: Polym. Chem., 37, 1017 (1999). 